\title{
The Baylis-Hillman reaction of substituted aminomethylbenzotriazoles
}

\author{
Alan R. Katritzky, ${ }^{\text {a* }}$ Myong Sang Kim, ${ }^{\text {a }}$ and Khalid Widyan ${ }^{\text {a, b }}$ \\ ${ }^{a}$ Center for Heterocyclic Compounds, Department of Chemistry, University of Florida \\ Gainesville, FL, 32611-7200, USA \\ ${ }^{b}$ Department of Chemistry, Tafila Technical University, P.O.Box 179, Tafila, Jordan \\ E-mail:katritzky@chem.ufl.edu
}

Dedicated to Professor Csaba Szantay on the occasion of his $80^{\text {th }}$ anniversary

\begin{abstract}
Aminomethylbenzotriazoles react as effective Baylis-Hillman electrophiles with ethyl acrylate in the presence of $\mathrm{TiCl}_{4}$ at $20{ }^{\circ} \mathrm{C}$ to afford the corresponding benzotriazolated adducts in $66-80 \%$ yield. These adducts are (i) readily transformed to the Baylis-Hillman olefins by sodium hydride and (ii) smoothly undergo substitution of Bt with thiols.
\end{abstract}

Keywords: Baylis-Hillman reaction, aminomethylbenzotriazoles, Baylis-Hillman olefins, substitution

\section{Introduction}

Carbon-carbon bond formation is the essence of organic synthesis and provides the foundation for generating more complicated organic compounds from simpler ones. Many classical carboncarbon bond-forming reactions are well documented e.g. the aldol, Friedel-Crafts, Reformatsky and Grignard reactions. More recently the Baylis-Hillman reaction, which couples electron deficient alkenes with carbon electrophiles has demonstrated great synthetic utility in converting simple starting materials into densely functionalized products, capable of numerous transformations. ${ }^{1,2}$

The Baylis-Hillman reaction had a precedent when in 1968, Morita described tricyclohexylphosphine catalyzed reactions of an aldehyde with acrylic compounds. ${ }^{3}$ In 1972 , Anthony Baylis and Melville Hillman patented catalyzed reactions using similar tertiary amine. ${ }^{4}$ In 1983, the reaction was rediscovered, and its scope was explored primarily by Drewes and Basavaiah. ${ }^{5}$ Since then, alkyl vinyl ketones, acrylonitriles, vinyl sulfones, acrylamides, allenic esters, vinyl sulfonates, vinyl sulfoxides, vinyl phosphonates, and acroleins have been substituted 
for the acrylate component. Imines, tosylimines, $\alpha$-ketoesters or aldehydes and fluoroesters have also been used. ${ }^{1}$

Versatile functionality, i.e. hydroxyl (or amino), alkene, and electron-withdrawing groups, make Baylis-Hillman adducts valuable intermediates in Friedel-Crafts ${ }^{6-8}$ and Heck reactions, ${ }^{9,10}$ hydride addition, ${ }^{11,12}$ hydrogenation, ${ }^{13-16}$ aminohydroxylation, ${ }^{17}$ radical $^{18,19}$ and photochemical reactions ${ }^{20}$ and 1,3-dipolar cycloaddition. ${ }^{21}$

Numerous chemical and physical methods have been developed ${ }^{22-27}$ to accelerate the BaylisHillman reaction, overcoming traditional slow reaction rates (weeks or months). ${ }^{28}$ Among Lewis acids, $\mathrm{TiCl}_{4}$ has been successfully utilized to promote the Baylis-Hillman reaction in the presence of Lewis base catalysts. Recent work has revealed that the combination of Lewis base such as phosphanes, ${ }^{29}$ organic chalcogenides, ${ }^{30-32}$ bisoxazolines ${ }^{33,34}$, amines ${ }^{35,36}$ and quaternary ammonium halides ${ }^{37,38}$ with $\mathrm{TiCl}_{4}$ can significantly improve the reaction rate. More interestingly, the combination of a Lewis base with the Lewis acid $\mathrm{TiCl}_{4}$ gives the corresponding chlorinated Baylis-Hillman products. ${ }^{35-38}$

Aminomethylbenzotriazoles $\mathbf{1}$ are useful synthetic intermediates ${ }^{39}$ in which the methylene carbon is highly electrophilic because of equilibrium with the benzotriazolide-iminium ion pair 2 (Scheme 1). ${ }^{40}$ Our group has successfully applied this concept to carbon-carbon bond formation reactions in their reactions with Grignard ${ }^{41,42}$ and Reformatsky ${ }^{43}$ reagents to provide secondary and tertiary amines. Aminomethylbenzotriazoles have been also successfully used for the synthesis of functionalized amides and $\beta$-aminoalkyl nitriles. ${ }^{44,45}$

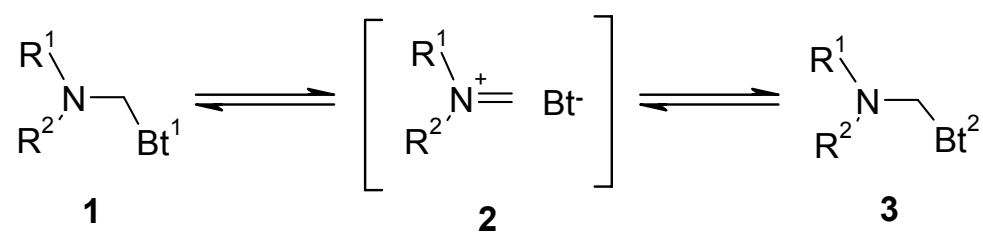

\section{Scheme 1}

We found only a single report of the Baylis-Hillman reaction using an iminium salt prepared in situ as an electrophile. ${ }^{46}$ The hygroscopicity, susceptibility to hydrolysis and the low stability of iminium salts with $\alpha-\mathrm{H}$ atoms ${ }^{47}$ hinder their isolation and encouraged coupling of electron deficient alkenes with the corresponding iminium salts bearing $\alpha-H$. We report herein reactions of aminomethylbenzotriazoles 1 with ethyl acrylate and analogs.

\section{Results and Discussion}

Aminomethylbenzotriazoles 1 are easily prepared according to well-established condensations of benzotriazole, formaldehyde and a secondary amine. ${ }^{48,49}$ 
Aminomethylbenzotriazoles 1 reacted with acrylates 4 in the presence of $\mathrm{TiCl}_{4}(1.0$ equiv) at $20{ }^{\circ} \mathrm{C}$ for $16-20 \mathrm{~h}$ to give exclusively the benzotriazolated product 5 in $66-80 \%$ yield rather than 6 which is usually expected as the product of the Baylis-Hillman reaction (Table 1). Extension of the reaction time to $72 \mathrm{~h}$ gave the same yields, but less than $12 \mathrm{~h}$ resulted in the isolation of the compound $\mathbf{5}$ in lower yield. To our knowledge, this is the first Baylis-Hillman reaction using iminium salts with $\alpha-\mathrm{H}$ atoms.

Table 1. Preparation of the Baylis-Hillman adducts 5

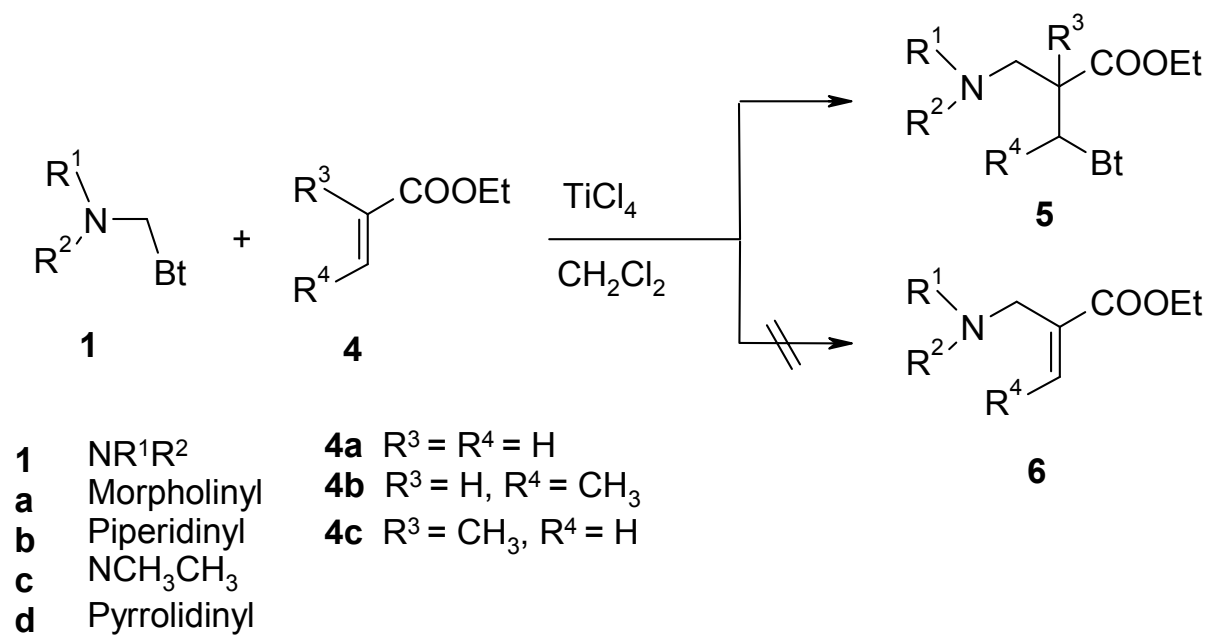

For designation of $\mathrm{R}^{1}, \mathrm{R}^{2}$ in $\mathbf{1}$ see Table 1

\begin{tabular}{|c|c|c|c|c|c|}
\hline 5 & $\mathbf{R}^{1}$ & $\mathbf{R}^{2}$ & $\mathbf{R}^{3}$ & $\mathbf{R}^{4}$ & Yield $(\%)$ \\
\hline $\mathbf{a}$ & \multicolumn{2}{|c|}{$\left(\mathrm{CH}_{2}\right)_{2} \mathrm{O}\left(\mathrm{CH}_{2}\right)_{2}$} & $\mathrm{H}$ & $\mathrm{H}$ & 69 \\
\hline b & \multicolumn{2}{|c|}{$\left(\mathrm{CH}_{2}\right)_{4}$} & $\mathrm{H}$ & $\mathrm{H}$ & 71 \\
\hline c & \multicolumn{2}{|c|}{$\left(\mathrm{CH}_{2}\right)_{5}$} & $\mathrm{H}$ & $\mathrm{H}$ & 73 \\
\hline d & \multicolumn{2}{|c|}{$\left(\mathrm{CH}_{2}\right)_{2} \mathrm{O}\left(\mathrm{CH}_{2}\right)_{2}$} & $\mathrm{H}$ & $\mathrm{CH}_{3}$ & 66 \\
\hline e & $\mathrm{CH}_{3}$ & $\mathrm{CH}_{3}$ & $\mathrm{H}$ & $\mathrm{H}$ & 80 \\
\hline f & \multicolumn{2}{|c|}{$\left(\mathrm{CH}_{2}\right)_{2} \mathrm{O}\left(\mathrm{CH}_{2}\right)_{2}$} & $\mathrm{CH}_{3}$ & $\mathrm{H}$ & 80 \\
\hline g & $\mathrm{CH}_{3}$ & $\mathrm{CH}_{3}$ & $\mathrm{CH}_{3}$ & $\mathrm{H}$ & 75 \\
\hline h & \multicolumn{2}{|c|}{$\left(\mathrm{CH}_{2}\right)_{4}$} & $\mathrm{CH}_{3}$ & $\mathrm{H}$ & 71 \\
\hline i & \multicolumn{2}{|c|}{$\left(\mathrm{CH}_{2}\right)_{5}$} & $\mathrm{CH}_{3}$ & $\mathrm{H}$ & 78 \\
\hline
\end{tabular}

Conversion of compounds 5a-d into the Baylis-Hillman olefin 7a-d was accomplished in 65$71 \%$ yields by treatment of $\mathbf{5 a - d}$ with sodium hydride in THF at room temperature for $5 \mathrm{~h}$. With one equivalent of sodium carbonate at $20{ }^{\circ} \mathrm{C}$, 5a gave 7a in low yield; a complex mixture was obtained under reflux conditions.

Compounds 7 are synthetic precursors for quaternary ammonium amphiphilic methacrylates, widely applied industrially, e.g., in waste-water treatment. ${ }^{50,51}$ Previous preparations of 7 from (i) 
mono ethyl malonate, formaldehyde, and a secondary amine ${ }^{52}$ or (ii) halomethyl acrylates and a secondary amine ${ }^{53,54}$ have several drawbacks such as tedious procedure, multi-step synthesis, slow reaction rates (days or weeks), and low yields and selectivity.

Table 2. Preparation of the Baylis-Hillman olefin 7

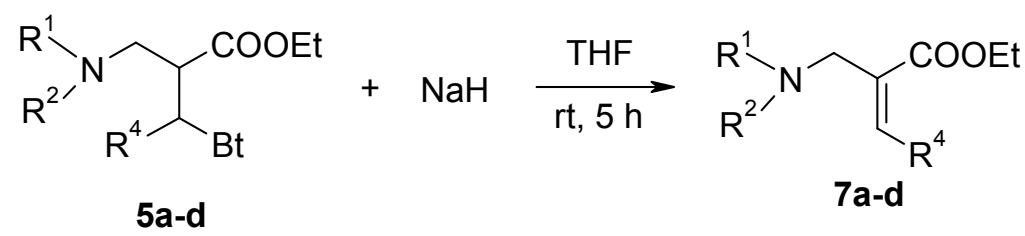

\begin{tabular}{ccccc}
\hline $\mathbf{7}$ & $\mathbf{R}^{\mathbf{1}}$ & $\mathbf{R}^{\mathbf{2}}$ & $\mathbf{R}^{\mathbf{4}}$ & Yield (\%) \\
\hline $\mathbf{a}$ & $\left(\mathrm{CH}_{2}\right)_{2} \mathrm{O}\left(\mathrm{CH}_{2}\right)_{2}$ & $\mathrm{H}$ & 71 \\
$\mathbf{b}$ & $\left(\mathrm{CH}_{2}\right)_{4}$ & $\mathrm{H}$ & 67 \\
c & $\left(\mathrm{CH}_{2}\right)_{5}$ & $\mathrm{H}$ & 68 \\
d & $\left(\mathrm{CH}_{2}\right)_{2} \mathrm{O}\left(\mathrm{CH}_{2}\right)_{2}$ & $\mathrm{CH}_{3}$ & 65 \\
\hline
\end{tabular}

Treatment of 5a-c with 2 equiv. of thiols in THF at $20-50{ }^{\circ} \mathrm{C}$ for the time indicated in Table 3 , monitoring the reactions by TLC, afforded novel compound 8a-g in 71-82\% yield (Table 3). The use of less than 2 equiv. of sodium thiolate left some unreacted starting material.

Table 3. Reaction of compounds 5 with thiols<smiles>[R7]CC(CN([R])[R])C(=O)OCC</smiles>

5a-c

$8 \mathrm{a}-\mathrm{g}$

\begin{tabular}{|c|c|c|c|c|c|}
\hline 8 & $\mathbf{R}^{2}$ & $\mathbf{R}^{5}$ & Temp & Time (h) & Yield (\%) \\
\hline $\mathbf{a}$ & $\left(\mathrm{CH}_{2}\right)_{2} \mathrm{O}\left(\mathrm{CH}_{2}\right)_{2}$ & $\mathrm{C}_{6} \mathrm{H}_{5}$ & RT & 12 & 82 \\
\hline b & $\left(\mathrm{CH}_{2}\right)_{2} \mathrm{O}\left(\mathrm{CH}_{2}\right)_{2}$ & 4- $\mathrm{CH}_{3} \mathrm{C}_{6} \mathrm{H}_{4}$ & RT & 12 & 79 \\
\hline c & $\left(\mathrm{CH}_{2}\right)_{2} \mathrm{O}\left(\mathrm{CH}_{2}\right)_{2}$ & $4-\mathrm{ClC}_{6} \mathrm{H}_{4}$ & reflux & 6 & 81 \\
\hline d & $\left(\mathrm{CH}_{2}\right)_{2} \mathrm{O}\left(\mathrm{CH}_{2}\right)_{2}$ & $\mathrm{C}_{2} \mathrm{H}_{5}$ & RT & 24 & 79 \\
\hline $\mathbf{e}$ & $\left(\mathrm{CH}_{2}\right)_{2} \mathrm{O}\left(\mathrm{CH}_{2}\right)_{2}$ & $4-\mathrm{CH}_{3} \mathrm{OC}_{6} \mathrm{H}_{4}$ & RT & 4 & 82 \\
\hline f & $\left(\mathrm{CH}_{2}\right)_{4}$ & $\mathrm{C}_{6} \mathrm{H}_{5}$ & RT & 12 & 71 \\
\hline g & $\left(\mathrm{CH}_{2}\right)_{5}$ & $\mathrm{C}_{6} \mathrm{H}_{5}$ & RT & 12 & 76 \\
\hline
\end{tabular}

The structures of all compounds 5, 7 and $\mathbf{8}$ were elucidated by ${ }^{1} \mathrm{H},{ }^{13} \mathrm{C}$ NMR spectroscopy and elemental analysis.

In summary we have developed a novel and general access to functionalized esters possessing an amino group at the $\beta$-position via the Baylis-Hillman reaction of ethyl acrylate and 
ethyl butenoate utilizing an easily accessible $N$-( $\alpha$-aminomethyl)benzotriazoles. The good yields of 5 demonstrate the convenience of $N$-( $\alpha$-aminomethyl)benzotriazoles as imminium ion equivalents with $\alpha-\mathrm{H}$ atoms generated in situ.

\section{Experimental Section}

General Procedures. Reagents were obtained form commercial suppliers and were used without further purification. All reactions were carried out under $\mathrm{N}_{2}$ atmosphere. ${ }^{1} \mathrm{H}$ NMR $(300 \mathrm{MHz})$ and ${ }^{13} \mathrm{C}(75 \mathrm{MHz})$ spectra were recorded on a $300 \mathrm{MHz}$ NMR spectrometer in $\mathrm{CDCl}_{3}$ using TMS as an internal standard. Column chromatography was performed on silica gel 200-425 mesh. THF was distilled from sodium-benzophenone ketyl and $\mathrm{CH}_{2} \mathrm{Cl}_{2}$ was distilled from $\mathrm{CaH}_{2}$ prior to use.

\section{General procedure for preparation of 5a-i}

$\mathrm{TiCl}_{4}(0.19 \mathrm{~g}, 1.0 \mathrm{mmol})$ was added to a solution of aminomethylbenzotriazole $(1.0 \mathrm{mmol})$ and acrylate $(2.0 \mathrm{mmol})$ in anhydrous $\mathrm{CH}_{2} \mathrm{Cl}_{2}(10 \mathrm{~mL})$ at $0{ }^{\circ} \mathrm{C}$. The resulting mixture was stirred at 0 ${ }^{\circ} \mathrm{C}$ for $30 \mathrm{~min}$ and room temperature for $16-20 \mathrm{~h}$. The mixture was quenched by saturated $\mathrm{NaHCO}_{3}(5 \mathrm{~mL})$. The organic phase was separated and the aqueous phase was extracted with ethyl acetate $(3 \times 10 \mathrm{~mL})$. The combined organic layers were washed with water and brine, dried over anhydrous $\mathrm{MgSO}_{4}$ and filtered. The solvent was evaporated under reduced pressure and the residue was purified by flash silica gel chromatography (eluent: diethyl ether $/ n$-hexane $=2 / 1$ )

Ethyl (3-benzotriazol-1-yl)-2-(morpholin-4-ylmethyl)propionate (5a). Yield 0.22 g (69\%); colorless oil; ${ }^{1} \mathrm{H}$ NMR $\delta 8.05(\mathrm{~d}, J=8.4 \mathrm{~Hz}, 1 \mathrm{H}), 7.58(\mathrm{~d}, J=8.4 \mathrm{~Hz}, 1 \mathrm{H}), 7.46-7.52(\mathrm{~m}, 1 \mathrm{H})$, 7.33-7.39 (m, $1 \mathrm{H}), 4.85-4.98(\mathrm{~m}, 2 \mathrm{H}), 3.96-4.10(\mathrm{~m}, 2 \mathrm{H}), 3.63(\mathrm{t}, J=4.2 \mathrm{~Hz}, 4 \mathrm{H}), 3.35-3.44(\mathrm{~m}$, $1 \mathrm{H}), 2.69(\mathrm{dd}, J=12.6,6.9 \mathrm{~Hz}, 1 \mathrm{H}), 2.60(\mathrm{dd}, J=12.6,8.5 \mathrm{~Hz}, 1 \mathrm{H}), 2.34-2.50(\mathrm{~m}, 4 \mathrm{H}), 1.08(\mathrm{t}, J$ $=7.1 \mathrm{~Hz}, 3 \mathrm{H}) ;{ }^{13} \mathrm{C}$ NMR $\delta 172.5,145.7,133.4,127.3,123.9,119.9,109.5,66.8,61.2,57.9,53.6$, 47.3, 44.0, 13.9; HRMS (ESI-TOF) : Calcd. for $\mathrm{C}_{16} \mathrm{H}_{22} \mathrm{~N}_{4} \mathrm{O}_{3}[\mathrm{M}+\mathrm{H}]^{+}$: 319.1771. Found: 319.1797

Ethyl (3-benzotriazol-1-yl)-2-(pyrrolidin-1-ylmethyl)propionate (5b). Yield 0.214 g (71\%); yellow oil; ${ }^{1} \mathrm{H}$ NMR $\delta 8.04(\mathrm{~d}, J=8.4 \mathrm{~Hz}, 1 \mathrm{H}), 7.60(\mathrm{~d}, J=8.4 \mathrm{~Hz}, 1 \mathrm{H}), 7.44-7.51(\mathrm{~m}, 1 \mathrm{H})$, 7.33-7.39 (m, 1H), 4.99 (dd, $J=14.0,5.4 \mathrm{~Hz}, 1 \mathrm{H}), 4.90(\mathrm{dd}, J=14.0,8.3 \mathrm{~Hz}, 1 \mathrm{H}), 3.95-4.08$ (m, 2H), 3.30-3.40 (m, 1H), 2.79 (dd, $J=11.2,6.8 \mathrm{~Hz}, 1 \mathrm{H}), 2.73(\mathrm{dd}, J=11.2,5.6 \mathrm{~Hz}, 1 \mathrm{H}), 2.40-$ $2.56(\mathrm{~m}, 4 \mathrm{H}), 1.72-1.81(\mathrm{~m}, 4 \mathrm{H}), 1.07(\mathrm{t}, J=7.1 \mathrm{~Hz}, 3 \mathrm{H}) ;{ }^{13} \mathrm{C}$ NMR $\delta 172.8,145.7,133.4,127.2$, 123.8, 119.8, 109.7, 61.1, 55.2, 54.1, 47.3, 46.1, 23.6, 13.9 Anal. Calcd. for $\mathrm{C}_{16} \mathrm{H}_{22} \mathrm{~N}_{4} \mathrm{O}_{2}$ : C, 63.55; H, 7.33; N, 18.53. Found: C, 63.66; H, 7.38; N, 18.26.

Ethyl (3-benzotriazol-1-yl)-2-(piperidin-1-ylmethyl)propionate (5c). Yield $0.23 \mathrm{~g}$ (73\%); pale yellow oil; ${ }^{1} \mathrm{H}$ NMR $\delta 8.04(\mathrm{~d}, J=8.4 \mathrm{~Hz}, 1 \mathrm{H}), 7.61(\mathrm{~d}, J=8.4 \mathrm{~Hz}, 1 \mathrm{H}), 7.45-7.51(\mathrm{~m}, 1 \mathrm{H})$, $7.32-7.39(\mathrm{~m}, 1 \mathrm{H}), 4.96(\mathrm{dd}, J=15.0,5.4 \mathrm{~Hz}, 1 \mathrm{H}), 4.88(\mathrm{dd}, J=15.0,8.1 \mathrm{~Hz}, 1 \mathrm{H}), 3.95-4.10(\mathrm{~m}$, 2H), 3.33-3.43 (m, 1H), 2.63 (dd, $J=12.0,6.5 \mathrm{~Hz}, 1 \mathrm{H}), 2.55(\mathrm{dd}, J=12.0,8.7 \mathrm{~Hz}, 1 \mathrm{H}), 2.30$ - 
$2.48(\mathrm{~m}, 4 \mathrm{H}), 1.48-1.59(\mathrm{~m}, 4 \mathrm{H}), 1.36-1.46(\mathrm{~m}, 2 \mathrm{H}), 1.08(\mathrm{t}, J=7.1 \mathrm{~Hz}, 3 \mathrm{H}) ; 13 \mathrm{C}$ NMR $\delta 172.8$, 145.5, 133.3, 127.1, 123.7, 119.6, 109.7, 60.9, 58.1, 54.5, 47.4, 44.3, 25.8, 24.1, 13.8 Anal. Calcd. for $\mathrm{C}_{17} \mathrm{H}_{24} \mathrm{~N}_{4} \mathrm{O}_{2}$ : C, 64.53; H, 7.65; N, 17.71. Found: C, 64.19; H, 7.68; N, 18.09

Ethyl (3-benzotriazol-1-yl)-3-methyl-2-(morpholin-4-ylmethyl)propionate (5d). Yield 0.218 $\mathrm{g}(66 \%)$; colorless oil; ${ }^{1} \mathrm{H}$ NMR $\delta 8.04(\mathrm{~d}, J=8.4 \mathrm{~Hz}, 1 \mathrm{H}), 7.58(\mathrm{~d}, J=8.4 \mathrm{~Hz}, 1 \mathrm{H}), 7.47-7.54$ $(\mathrm{m}, 1 \mathrm{H}), 7.36-7.43(\mathrm{~m}, 1 \mathrm{H}), 5.10-5.22(\mathrm{~m}, 1 \mathrm{H}), 4.25(\mathrm{q}, J=7.2 \mathrm{~Hz}, 2 \mathrm{H}), 3.50-3.58(\mathrm{~m}, 1 \mathrm{H})$, 3.37-3.46 (m, 4H), 2,55 (dd, $J=159.2 \mathrm{~Hz}, 1 \mathrm{H}), 2.22-2.32(\mathrm{~m}, 2 \mathrm{H}), 2.01-2.12(\mathrm{~m}, 3 \mathrm{H}), 1.73(\mathrm{~d}$, $J=6.9 \mathrm{~Hz}, 3 \mathrm{H}), 1.30(\mathrm{t}, J=7.2 \mathrm{~Hz}, 3 \mathrm{H}) ;{ }^{13} \mathrm{C} \mathrm{NMR} \delta 172.6,145.9,132.8,127.3,124.0,120.1$, 109.3, 66.6, 61.0, 58.4, 55.5, 53.4, 49.3, 19.4, 14.2 Anal. Calcd. for $\mathrm{C}_{17} \mathrm{H}_{24} \mathrm{~N}_{4} \mathrm{O}_{23}$ : C, 61.43; $\mathrm{H}$, 7.28; N, 16.85. Found: C, 61.79; H, 7.49; N, 16.22.

Ethyl (3-benzotriazol-1-yl)-2-( $\boldsymbol{N}, \boldsymbol{N}$-dimethylaminomethyl)propionate (5e). Yield $0.22 \mathrm{~g}$ (80\%); colorless oil; ${ }^{1} \mathrm{H}$ NMR $\delta(\mathrm{d}, J=8.4 \mathrm{~Hz}, 1 \mathrm{H}), 7.60(\mathrm{~d}, J=8.4 \mathrm{~Hz}, 1 \mathrm{H}), 7.46-7.52(\mathrm{~m}, 1 \mathrm{H})$, 7.33-7.39 (m, 1H), $4.94(\mathrm{dd}, J=14.2,5.4 \mathrm{~Hz}, 1 \mathrm{H}), 4.87(\mathrm{dd}, J=14.2,8.3 \mathrm{~Hz}, 1 \mathrm{H}), 3.96-4.06(\mathrm{~m}$, $2 \mathrm{H}), 3.29-3.38(\mathrm{~m}, 1 \mathrm{H}), 2.64(\mathrm{dd}, J=12.5,6.8 \mathrm{~Hz}, 1 \mathrm{H}), 2.54(\mathrm{dd}, J=12.5,8.3 \mathrm{~Hz}, 1 \mathrm{H}), 2.27(\mathrm{~s}$, $6 \mathrm{H}), 1.04(\mathrm{t}, J=7.1 \mathrm{~Hz}, 3 \mathrm{H}) ;{ }^{13} \mathrm{C}$ NMR $\delta 172.7,145.7,133.3,127.3,123.8,119.8,109.6,61.1$, 58.8, 47.3, 45.6, 45.1, 13.8; HRMS (ESI-TOF) : Calcd. for $\mathrm{C}_{14} \mathrm{H}_{20} \mathrm{~N}_{4} \mathrm{O}_{2}[\mathrm{M}+\mathrm{H}]^{+}: 277.1665$. Found 277.1689 .

Ethyl (3-benzotriazol-1-yl)-2-methyl-2-(morphlinylmethyl)propionate (5f). Yield $0.264 \mathrm{~g}$ (80\%); pale yellow oil; ${ }^{1} \mathrm{H}$ NMR $\delta 8.05(\mathrm{~d}, J=8.4 \mathrm{~Hz}, 1 \mathrm{H}), 7.63(\mathrm{~d}, J=8.4 \mathrm{~Hz}, 1 \mathrm{H}), 7.45-7.52$ (m, 1H), 7.33-7.39 (m, 1H), $4.91(\mathrm{~s}, 2 \mathrm{H}), 4.12$ (dd, J=7.2, 2.7 Hz. 1H), 4.07 (dd, J = 7.2, $2.4 \mathrm{~Hz}$, $1 \mathrm{H}), 3.69(\mathrm{t}, J=4.7 \mathrm{~Hz}, 4 \mathrm{H}), 2.71(\mathrm{~d}, J=13.8 \mathrm{~Hz}, 1 \mathrm{H}$, A part of AB system), $2.65(\mathrm{~d}, J=13.8$ $\mathrm{Hz}, 1 \mathrm{H}, \mathrm{B}$ part of AB system), $2.54(\mathrm{t}, J=4.7 \mathrm{~Hz}, 4 \mathrm{H}), 1.22(\mathrm{~s}, 3 \mathrm{H}), 1.17(\mathrm{t}, J=7.1 \mathrm{~Hz}, 3 \mathrm{H}) ;{ }^{13} \mathrm{C}$ NMR $\delta 175.0,145.4,134.1,127.2,123.9,119.8,110.1,67.1,63.5,61.2,55.1,51.6,48.7,20.3$, 13.9 Anal. Calcd. for $\mathrm{C}_{17} \mathrm{H}_{24} \mathrm{~N}_{4} \mathrm{O}_{3}$ : C, 61.43; H, 7.28; N, 16.85. Found: C, 61.13; H, 7.33; N, 17.26

Ethyl (3-benzotriazol-1-yl)-2-( $\mathbf{N}, \boldsymbol{N}$-dimethylaminomethyl)-2-methylpropionate (5g). Yield $0.218 \mathrm{~g}(75 \%)$; pale yellow oil; $1 \mathrm{H}$ NMR $\delta 8.04(\mathrm{~d}, J=8.4 \mathrm{~Hz}, 1 \mathrm{H}), 7.66(\mathrm{~d}, J=8.4 \mathrm{~Hz}, 1 \mathrm{H})$, 7.44-7.50 (m, 1H), 7.32-7.38 (m, 1H), $4.93(\mathrm{~d}, J=14.3 \mathrm{~Hz}, 1 \mathrm{H}$, A part of AB system), $4.86(\mathrm{~d}, J$ $=14.3 \mathrm{~Hz}, 1 \mathrm{H}, \mathrm{B}$ part of AB system), $4.14(\mathrm{dd}, J=7.2,1.5 \mathrm{~Hz}, 1 \mathrm{H}), 4.09(\mathrm{dd}, J=7.2,1.5 \mathrm{~Hz}$, $1 \mathrm{H}), 2.64(\mathrm{~d}, J=13.5 \mathrm{~Hz}, 1 \mathrm{H}, \mathrm{A}$ part of AB system), $2.58(\mathrm{~d}, J=13.5 \mathrm{~Hz}, 1 \mathrm{H}, \mathrm{B}$ part of AB system), $2.27(\mathrm{~s}, 6 \mathrm{H}), 1.22(\mathrm{~s}, 3 \mathrm{H}), 1.18(\mathrm{t}, J=7.1 \mathrm{~Hz}, 3 \mathrm{H}) ;{ }^{13} \mathrm{C}$ NMR $\delta 175.1,145.4,134.2$, 127.1, 123.7, 119.7, 110.4, 64.9, 61.2, 51.8, 48.7, 47.3, 20.3, 13.9 Anal. Calcd. for $\mathrm{C}_{15} \mathrm{H}_{22} \mathrm{~N}_{4} \mathrm{O}_{2}$ : C, 62.05; H, 7.64; N, 19.30. Found: C, 62.39; H, 7.86; N, 18.92

Ethyl (3-benzotriazol-1-yl)-2-methyl-2-(pyrrodin-1-ylmethyl)propionate (5h). Yield $0.224 \mathrm{~g}$ (71\%); pale yellow oil; 1H NMR $\delta 8.04(\mathrm{~d}, J=8.4 \mathrm{~Hz}, 1 \mathrm{H}), 7.69(\mathrm{~d}, J=8.4 \mathrm{~Hz}, 1 \mathrm{H}), 7.43-7.48$ $(\mathrm{m}, 1 \mathrm{H}), 7.31-7.37(\mathrm{~m}, 1 \mathrm{H}), 4.95(\mathrm{~d}, J=14.4 \mathrm{~Hz}, 1 \mathrm{H}$, A part of AB system), $4.91(\mathrm{~d}, J=14.4 \mathrm{~Hz}$, $1 \mathrm{H}, \mathrm{B}$ part of AB system), $4.16(\mathrm{dd}, J=7.2,2.4 \mathrm{~Hz}, 1 \mathrm{H}), 4.11(\mathrm{dd}, J=7.2,2.1 \mathrm{~Hz}, 1 \mathrm{H}), 2.75(\mathrm{~d}$, $J=13.2 \mathrm{~Hz}, 1 \mathrm{H}$, A part of AB system), 2.67 (d, $J=13.2 \mathrm{~Hz}, 1 \mathrm{H}, \mathrm{B}$ part of AB system), 2.50$2.59(\mathrm{~m}, 2 \mathrm{H}), 2.43-2.49(\mathrm{~m}, 2 \mathrm{H}), 1.70-1.79(\mathrm{~m}, 4 \mathrm{H}), 1.27(\mathrm{~s}, 3 \mathrm{H}), 1.21(\mathrm{t}, \mathrm{J}=7.2 \mathrm{~Hz}, 3 \mathrm{H}) ;{ }^{13} \mathrm{C}$ NMR $\delta 175.4,145.4,134.2,126.9,123.6,119.6,110.5,61.5,61.1,55.3,51.6,48.6,23.9,20.8$, 
13.9 Anal. Calcd. for $\mathrm{C}_{17} \mathrm{H}_{24} \mathrm{~N}_{4} \mathrm{O}_{2}$ : C, 64.53; H, 7.65; N, 17.71. Found: C, 64.84; H, 7.65; N, 17.23

Ethyl (3-benzotriazol-1-yl)-2-methyl-2-(piperidin-1-ylmethyl)propionate (5i). Yield $0.257 \mathrm{~g}$ (78\%); pale yellow oil; 1H NMR $\delta 8.04(\mathrm{~d}, J=8.4 \mathrm{~Hz}, 1 \mathrm{H}), 7.68(\mathrm{~d}, J=8.4 \mathrm{~Hz}, 1 \mathrm{H}), 7.44-7.50$ $(\mathrm{m}, 1 \mathrm{H}), 7.32-7.37(\mathrm{~m}, 1 \mathrm{H}), 4.94(\mathrm{~d}, J=14.3 \mathrm{~Hz}, 1 \mathrm{H}, \mathrm{A}$ part of AB system), $4.84(\mathrm{~d}, J=14.3 \mathrm{~Hz}$, $1 \mathrm{H}$, B part of AB system), 4.09 (q, $J=7.2 \mathrm{~Hz}, 2 \mathrm{H}), 2.65(\mathrm{~d}, J=13.8 \mathrm{~Hz}, 1 \mathrm{H}, \mathrm{A}$ part of AB system), $2.56(\mathrm{~d}, J=13.8 \mathrm{~Hz}, 1 \mathrm{H}, \mathrm{B}$ part of AB system), 2.38-2.50 (m, 4H), 1.50-1.59 (m, 4H), 1.40-1.43 (m, 2H), 1.21 (s, 3H), 1.17 (t, $J=7.2 \mathrm{~Hz}, 3 \mathrm{H})$; 13C NMR $\delta 175.2,145.4,134.1,127.0$, 123.7, 119.7, 110.4, 64.1, 61.0, 56.2, 51.9, 48.9, 26.3, 23.9, 20.0, 13.9 Anal. Calcd. for $\mathrm{C}_{18} \mathrm{H}_{26}$ $\mathrm{N}_{4} \mathrm{O}_{2}: \mathrm{C}, 65.43 ; \mathrm{H}, 7.93 ; \mathrm{N}, 16.96$. Found: C, 65.04; H, 8.01; N, 16.76

\section{General procedure for preparation of compound 7a-d}

Compound $5(1.0 \mathrm{mmol})$ dissolved in THF $(1 \mathrm{~mL})$ was added dropwise to a suspension of sodium hydride $(0.024 \mathrm{~g}, 1 \mathrm{mmol}, 60 \%$ dispersed in mineral oil) in anhydrous THF (5 mL) and the mixture was stirred at room temperature for $5 \mathrm{~h}$. The resulting mixture was quenched by saturated $\mathrm{NaCl}$ solution, extracted with diethyl ether $(2 \times 15 \mathrm{~mL})$. The organic phase was dried over anhydrous $\mathrm{MgSO}_{4}$ and solvent was evaporated under reduced pressure. The residue was purified by flash silica gel chromatography (eluent: $n$-hexane/diethyl ether $=2 / 1$ ).

Ethyl 2-(morphlinomethyl)acrylate (7a). ${ }^{52-54}$ Yield $0.140 \mathrm{~g}(71 \%)$; yellow oil; ${ }^{1} \mathrm{H}$ NMR $\delta 6.27$ $(\mathrm{d}, J=0.6 \mathrm{~Hz}, 1 \mathrm{H}), 5.76(\mathrm{~d}, J=1.5 \mathrm{~Hz}, 1 \mathrm{H}), 4.22$ (q, $J=7.2 \mathrm{~Hz}, 2 \mathrm{H}), 3.70$ (t, $J=4.7 \mathrm{~Hz}, 4 \mathrm{H})$, $3.20(\mathrm{~s}, 2 \mathrm{H}), 2.47(\mathrm{t}, J=4.7 \mathrm{~Hz}, 4 \mathrm{H}), 1.31(\mathrm{t}, J=7.2 \mathrm{~Hz}, 3 \mathrm{H}) ;{ }^{13} \mathrm{C}$ NMR $\delta 166.8,136.8,126.5$, $67.0,60.7,58.8,53.5,14.1$.

Ethyl 2-(pyrolidin-1-ylmethyl)acrylate (7b). ${ }^{52-54}$ Yield 0.123 g (67\%); yellow oil; ${ }^{1} \mathrm{H}$ NMR $\delta 6.27(\mathrm{~s}, 1 \mathrm{H}), 5.78(\mathrm{~d}, J=1.5 \mathrm{~Hz}, 1 \mathrm{H}), 4.22(\mathrm{q}, J=7.2 \mathrm{~Hz}, 2 \mathrm{H}), 3.34(\mathrm{~s}, 2 \mathrm{H}), 2.50-2.57$ (m, 4H), 1.74-1.81 (m, 4H), $1.31(\mathrm{t}, J=7.2 \mathrm{~Hz}, 3 \mathrm{H}) ;{ }^{13} \mathrm{C} \mathrm{NMR} \delta 166.9,138.2,126.1,60.6,56.1,54.1$, 23.5, 14.2 .

Ethyl 2-(piperdin-1-ylmethyl)acrylate (7c). ${ }^{52-54}$ Yield $0.134 \mathrm{~g}(68 \%)$; yellow oil; ${ }^{1} \mathrm{H}$ NMR $\delta$ $(\mathrm{m}, 1 \mathrm{H}), 5.75(\mathrm{~d}, J=1.8 \mathrm{~Hz}, 1 \mathrm{H}), 4.23$ (q, $J=7.2 \mathrm{~Hz}, 2 \mathrm{H}), 3.17$ (s, 2H), 2.36-2.43 (m, 4H), 1.53$1.61(\mathrm{~m}, 4 \mathrm{H}), 1.38-1.46(\mathrm{~m}, 2 \mathrm{H}), 1.30(\mathrm{t}, J=7.2 \mathrm{~Hz}, 3 \mathrm{H}) ;{ }^{13} \mathrm{C}$ NMR $\delta 167.1,137.4,126.1,60.5$, $58.9,54.5,26.0,24.3,14.2$.

Ethyl 2-(morpholinomethyl)but-2-enoate (7d). Yield $0.138 \mathrm{~g}(65 \%)$; yellow oil; ${ }^{1} \mathrm{H}$ NMR $\delta$ 7.02 (q, $J=7.2 \mathrm{~Hz}, 1 \mathrm{H}), 4.20(\mathrm{q}, J=7.2 \mathrm{~Hz}, 2 \mathrm{H}), 3.67$ (t, $J=4.7 \mathrm{~Hz}, 4 \mathrm{H}), 3.25$ (s, 2H), 2.44 (t, $J=4.7 \mathrm{~Hz}, 4 \mathrm{H}), 1.89(\mathrm{~d}, J=7.2 \mathrm{~Hz}, 3 \mathrm{H}), 1.29(\mathrm{t}, J=7.1 \mathrm{~Hz}, 3 \mathrm{H}) ;{ }^{13} \mathrm{C} \mathrm{NMR} \delta 167.7,141.0$, 129.7, 67.0, 60.4, 53.3, 53.0, 14.7, 14.2. Anal. Calcd. for C11H19NO3: C, 61.95; H, 8.98; N, 6.57. Found: C, 62.24; H, 9.35; N, 6.58.

\section{General procedure for the synthesis of compound 8a-g}

Sodium hydride $(0.055 \mathrm{~g}, 2.3 \mathrm{mmol}, 60 \%$ dispersed in mineral oil) was added to solution of thiol $(2.2 \mathrm{mmol})$ in anhydrous THF $(5 \mathrm{~mL})$ at room temperature, and the reaction mixture was stirred for $1 \mathrm{~h}$. Compound $5(1.0 \mathrm{mmol})$ dissolved in THF $(1 \mathrm{~mL})$ was added dropwise at room 
temperature, the reaction mixture was stirred at room temperature or under reflux for the time indicated in Table 3. The resulting mixture was quenched by saturated $\mathrm{NaCl}$ solution, extracted with diethyl ether $(2 \times 15 \mathrm{~mL})$. The organic phase was dried over anhydrous $\mathrm{MgSO}_{4}$ and solvent was evaporated under reduced pressure. The residue was purified by flash silica gel chromatography (eluent: $n$-hexane/diethyl ether $=2 / 1$ ).

Ethyl 2-(morpholin-4-ylmethyl)-3-phenylthiopropionate (8a). Yield $0.253 \mathrm{~g}(82 \%)$; colorless oil; ${ }^{1} \mathrm{H}$ NMR $\delta$ 7.37-7.41 (m, 2H), 7.25-7.32 (m, 2H), 7.16-7.23 (m, 1H), $4.16(\mathrm{dd}, J=7.2,1.2$ $\mathrm{Hz}, 1 \mathrm{H}), 4.11$ (dd, $J=7.2,1.2 \mathrm{~Hz}, 1 \mathrm{H}), 3.64(\mathrm{t}, J=4.7 \mathrm{~Hz}, 4 \mathrm{H}), 3.16$ (d, $J=6.6 \mathrm{~Hz}, 2 \mathrm{H}), 2.83$ (qn, $J=7.2 \mathrm{~Hz}, 1 \mathrm{H}$ ), 2.63 (dd, $J=12.3,7.2 \mathrm{~Hz}, 1 \mathrm{H}$ ), 2.52, (dd, $J=12.3,7.2 \mathrm{~Hz}, 1 \mathrm{H}$ ), 2.32-2.45 $(\mathrm{m}, 4 \mathrm{H}), 1.25(\mathrm{t}, J=7.1 \mathrm{~Hz}, 3 \mathrm{H}) ;{ }^{13} \mathrm{C}$ NMR $\delta 173.5,135.7,130.0,128.9,126.4,66.9,60.7,59.7$, 53.6, 43.8, 33.9, 14.2. Anal. Calcd. for $\mathrm{C}_{16} \mathrm{H}_{23} \mathrm{NO}_{3} \mathrm{~S}: \mathrm{C}, 62.11 ; \mathrm{H}, 7.49 ; \mathrm{N}, 4.53$. Found: $\mathrm{C}, 62.40$; $\mathrm{H}, 7.49 ; \mathrm{N}, 4.68$.

Ethyl 3-(4-methylphenylthio)-2-(morpholin-4-ylmethyl)propionate (8b). Yield $0.255 \mathrm{~g}$ (79\%); yellow oil; ${ }^{1} \mathrm{H}$ NMR $\delta 7.29(\mathrm{~d}, J=8.0 \mathrm{~Hz}, 2 \mathrm{H}), 7.10(\mathrm{~d}, J=8.0 \mathrm{~Hz}, 2 \mathrm{H}), 4.16$ (dd, $J=9.0$, $0.9 \mathrm{~Hz}, 1 \mathrm{H}), 4.11(\mathrm{dd}, J=9.0,0.9 \mathrm{~Hz}, 1 \mathrm{H}), 3.63(\mathrm{t}, J=4.8 \mathrm{~Hz}, 4 \mathrm{H}), 3.10(\mathrm{~d}, J=6.6 \mathrm{~Hz}, 2 \mathrm{H})$, 2.81 (qn, $J=4.2 \mathrm{~Hz}, 1 \mathrm{H}), 2.61$, (dd, $J=12.0,7.8 \mathrm{~Hz}, 1 \mathrm{H}), 2.50$ (dd, $J=12.0,7.2 \mathrm{~Hz}, 1 \mathrm{H}$ ), 2.34$2.44(\mathrm{~m}, 4 \mathrm{H}), 2.32(\mathrm{~s}, 3 \mathrm{H}), 1.25(\mathrm{t}, J=7.2 \mathrm{~Hz}, 3 \mathrm{H}) ;{ }^{13} \mathrm{C}$ NMR $\delta$ 173.6, 136.6, 131.8, 130.8, 129.7, 66.9, 60.6, 59.7, 53.6, 43.7, 34.6, 21.0, 14.2. Anal. Calcd. for $\mathrm{C}_{17} \mathrm{H}_{25} \mathrm{NO}_{3} \mathrm{~S}: \mathrm{C}, 63.13 ; \mathrm{H}, 7.79 ; \mathrm{N}$, 4.33. Found: C, 63.28; H, 7.86; N, 4.41.

Ethyl 3-(4-chlorophenylthio)-2-(morpholin-4-ylmethyl)propionate (8c). Yield $0.278 \mathrm{~g} \mathrm{(81 \% );}$ colorless oil; ${ }^{1} \mathrm{H}$ NMR $\delta$ 7.29-7.34 (m, 2H), 7.23-7.28 (m, $\left.2 \mathrm{H}\right), 4.16(\mathrm{dd}, J=7.1,1.8 \mathrm{~Hz}, 1 \mathrm{H})$, 4.11 (dd, $J=7.1,1.7 \mathrm{~Hz}, 1 \mathrm{H}), 3.64$ (t, $J=4.7 \mathrm{~Hz}, 4 \mathrm{H}), 3.14$ (d, $J=6.6 \mathrm{~Hz}, 2 \mathrm{H}), 2.81$ (qn, $J=7.2$ $\mathrm{Hz}, 1 \mathrm{H}), 2.62$ (dd, $J=12.3,7.5 \mathrm{~Hz}, 1 \mathrm{H}), 2.50$ (dd, $J=12.3,7.5 \mathrm{~Hz}, 1 \mathrm{H}), 2.33-2.46$ (m, 4H), 1.25 (t, $J=7.2 \mathrm{~Hz}, 3 \mathrm{H}) ;{ }^{13} \mathrm{C}$ NMR $\delta 173.3,134.2,132.5,131.3,129.0,66.9,60.8,59.7,53.6,43.7$, 34.1, 14.2. Anal. Calcd. for $\mathrm{C}_{16} \mathrm{H}_{22} \mathrm{ClNO}_{3} \mathrm{~S}: \mathrm{C}, 55.88 ; \mathrm{H}, 6.45 ; \mathrm{N}, 4.07$. Found: $\mathrm{C}, 56.28 ; \mathrm{H}, 6.66$; N, 4.28 .

Ethyl 3-ethylthio-2-(morpholin-4-ylmethyl)propionate (8d). Yield $0.206 \mathrm{~g} \mathrm{(79 \% );} \mathrm{colorless}$ oil; ${ }^{1} \mathrm{H}$ NMR $\delta 4.21(\mathrm{dd}, J=7.2,1.2 \mathrm{~Hz}, 1 \mathrm{H}), 4.17(\mathrm{dd}, J=7.2,1.2 \mathrm{~Hz}, 1 \mathrm{H}), 3.66(\mathrm{t}, J=4.7 \mathrm{~Hz}$, 4H), 2.78-2.87 (m, 1H), 2.74 (dd, $J=6.9,3.3 \mathrm{~Hz}, 2 \mathrm{H}), 2.55-2.68(\mathrm{~m}, 2 \mathrm{H}), 2.45-2.53(\mathrm{~m}, 4 \mathrm{H})$, 2.37-2.44 (m, 2H), 1.21-1.31 (m, 6H); ${ }^{13} \mathrm{C}$ NMR $\delta 173.9,66.9,60.5,60.0,53.6,44.2,31.5,26.4$, 14.6, 14.3. Anal. Calcd. for $\mathrm{C}_{12} \mathrm{H}_{23} \mathrm{NO}_{3} \mathrm{~S}$ : C, 55.14; H, 8.87; N, 5.36. Found: C, 55.10; H, 9.24; N, 5.16 .

Ethyl 3-(4-methoxyphenylthio)-2-(morpholin-4-ylmethyl)propionate (8e). Yield $0.278 \mathrm{~g}$ (82\%); yellow oil; ${ }^{1} \mathrm{H}$ NMR $\delta$ 7.36-7.41 (m, $\left.2 \mathrm{H}\right), 6.82-6.86(\mathrm{~m}, 2 \mathrm{H}), 4.16(\mathrm{dd}, J=6.0,1.2 \mathrm{~Hz}$, $1 \mathrm{H}), 4.11(\mathrm{dd}, J=6.0,0.9 \mathrm{~Hz}, 1 \mathrm{H}), 3.80(\mathrm{~s}, 3 \mathrm{H}), 3.62(\mathrm{t}, J=4.7 \mathrm{~Hz}, 4 \mathrm{H}), 3.00-3.09(\mathrm{~m}, 2 \mathrm{H})$, 2.73-2.83 (m, 1H), $2.60(\mathrm{dd}, J=12.0,8.1 \mathrm{~Hz}, 1 \mathrm{H}), 2.48(\mathrm{dd}, J=12.0,6.9 \mathrm{~Hz}, 1 \mathrm{H}), 2.28-2.44(\mathrm{~m}$, $4 \mathrm{H}), 1.26(\mathrm{t}, J=7.1 \mathrm{~Hz}, 3 \mathrm{H}) ;{ }^{13} \mathrm{C}$ NMR $\delta 173.7,159.2,133.9,125.6,114.5,66.9,60.6,59.7$, 55.3, 53.6, 43.8, 36.0, 14.3. Anal. Calcd. for $\mathrm{C}_{17} \mathrm{H}_{25} \mathrm{NO}_{4} \mathrm{~S}: \mathrm{C}, 60.15 ; \mathrm{H}, 7.42 ; \mathrm{N}, 4.13$. Found: $\mathrm{C}$, $60.43 ; \mathrm{H}, 7.66 ; \mathrm{N}, 4.27$. 
Ethyl 3-phenylthio-2-(pyrrolidin-1-ylmethyl)propionate (8f). Yield 0.208 g (71\%); yellow oil; ${ }^{1} \mathrm{H}$ NMR $\delta$ 7.36-7.41 (m, 2H), 7.26-7.30 (m, 2H), 7.16-7.22 (m, 1H), 4.13 (q, J=7.2 Hz, 2H), 3.11-3.24 (m, 2H), 2.77-2.86 (m, 1H), 2.64-2.75 (m, 2H), 2.40-2.48 (m, 4H), 1.68-1.76 (m, 4H), $1.24(\mathrm{t}, J=7.2 \mathrm{~Hz}, 3 \mathrm{H})$; 13C NMR $\delta 173.8,135.9,123.0,128.8,126.3,60.6,57.3,54.1,45.8$, 34.2, 23.6, 14.2 Anal. Calcd. for $\mathrm{C}_{16} \mathrm{H}_{23} \mathrm{NO}_{2} \mathrm{~S}$ : C, 65.49; H, 7.90; N, 4.77. Found: C, 65.47; H, 8.04; N, 5.05 .

Ethyl 3-phenylthio-2-(piperidin-1-ylmethyl)propionate (8g). Yield 0.233 g (76\%); yellow oil; ${ }^{1} \mathrm{H}$ NMR $\delta$ 7.35-7.41 (m, 2H), 7.24-7.32 (m, 2H), 7.15-7.21 (m, 1H), $4.12(\mathrm{q}, J=7.2 \mathrm{~Hz}, 2 \mathrm{H})$, $3.22(\mathrm{dd}, J=13.2,4.8 \mathrm{~Hz}, 1 \mathrm{H}$, A part of AB system), $3.13(\mathrm{dd}, J=13.2,8.7 \mathrm{~Hz}, 1 \mathrm{H}$, B part of AB system), 2.80-2.89 (m, 1H), 2.57 (dd, $J=12.4,7.2 \mathrm{~Hz}, 1 \mathrm{H}$, A part of AB system), 2.47 (dd, $J$ $=12.4,7.7 \mathrm{~Hz}, 1 \mathrm{H}, \mathrm{B}$ part of $\mathrm{AB}$ system $), 2.27-2.37(\mathrm{~m}, 4 \mathrm{H}), 1.46-1.56(\mathrm{~m}, 4 \mathrm{H}), 1.35-1.43(\mathrm{~m}$, 2H), 1.24 (t, $J=7.2 \mathrm{~Hz}, 3 \mathrm{H})$; 13C NMR $\delta 173.9,136.0,129.6,128.8,126.1,60.5,60.2,54.7$, 44.2, 33.8, 26.0, 24.3, 14.2 Anal. Calcd. for $\mathrm{C}_{17} \mathrm{H}_{25} \mathrm{NO}_{2} \mathrm{~S}$ : C, 66.41; H, 8.20; N, 4.56. Found: C, $66.73 ; \mathrm{H}, 8.41 ; \mathrm{N}, 4.44$.

\section{References and Notes}

1. Basavaiah, D.; Rao, A. J.; Satanarayana, T. Chem. Rev. 2003, 103, 811 and references therein.

2. Lee, K. Y.; Gowrisankar, S.; Kim, J. N. Bull. Korean Chem. Soc. 2005, 26, 1481 and reference therein.

3. Morita, K.; Suzuki, Z.; Hirose, H. Bull. Chem. Soc. Jpn. 1968, 41, 2815.

4. Baylis, A. B.; Hillman, M. E. D. German Patent 2155 113, 1972; Chem. Abstr. 1972, 77, 34174q.

5. Drewes, S. E.; Roos, G. H. P. Tetrahedron 1988, 44, 4653.

6. Basavaiah, D.; Krishnamacharyulu, M.; Hyma, R.S.; Pandiaraju, S. Tetrahedron Lett. 1997, $38,2141$.

7. Lee, H. J.; Seong, M. R.; Kim, J. N. Tetrahedron Lett. 1998, 39, 6223.

8. Basavaiah, D.; Bakthadoss, M.; Pandiaraju, S. Chem. Commun. 1998, 1639.

9. Calo, V.; Nacci, A.; Lopez, L.; Napola, A. Tetrahedron 2001, 42, 4703

10. Basavaiah, D.; Muthukumaran, K. Tetrahedron 1998, 54, 4943.

11. Pachamuthu, K.; Vankar, Y. D. Tetrahedron Lett. 1998, 39, 5439.

12. Shadakshari, U.; Nayak, S. K. Tetrahedron 2001, 57, 4599.

13. Kitamura, M.; Kasahara, I.; Manabe, K.; Noyori, R.; Takaya, H. J. Org. Chem. 1988, 53, 708.

14. Farrington, E.; Franchini, M. C.; Brown, J. M. Chem. Commun. 1998, 277.

15. Bouzide, A. Org. Lett. 2002, 4, 1347.

16. Coelho, F.; Almeida, W. P.; Mateus C. R.; Furtado, L. D.; Gouveia, J. C. F. ARKIVOC 2003, $(x), 443$. 
17. Pringle, W.; Sharpless, K. B. Tetrahedron Lett. 1999, 40, 5151.

18. Guindon, Y.; Rancourt, J. J. Org. Chem. 1998, 63, 6554.

19. Mase, N.; Watanabe, Y.; Toru, T. Tetrahedron Lett. 1999, 40, 2797.

20. Matsumoto, S.; Okubo, Y.; Mikami, K. J. Am. Chem. Soc. 1998, 120, 4015.

21. Dugovič, B.; Fišera, L.; Hametner, C.; Prónayová, N. ARKIVOC 2003, (xiv), 162.

22. Yu, C.; Liu, B.; Hu, L. J. Org. Chem. 2001, 66, 5413.

23. Lee, W-D; Yang, K-S; Chen, K. Chem. Commun. 2001, 1612.

24. Aggarwal, V. K.; Dean, D. K.; Mereu, A.; Williams, R. J. Org. Chem. 2002, 67, 510.

25. Yu, C.; Hu, L. J. Org. Chem. 2002, 67, 219.

26. Basavaiah, D.; Rao, A. J.; Krishnamacharyulu, M. ARKIVOC 2002, (vii), 136.

27. Krishna, P. R.; Manjuvani, A.; Sekhar, E. R. ARKIVOC 2005, (iii), 99.

28. Langer, P. Angew. Chem. Int. Ed. 2000, 39, 3409 and references therein.

29. You, J.; Xu, J.; Verkade, J. G. Angew. Chem. Int. Ed. 2003, 42, 5054.

30. Kataoka, T.; Kinoshita, H. Eur. J. Org. Chem. 2005, 45 and references therein

31. Shi, M.; Jiang, J-K. Tetrahedron 2000, 56, 4793.

32. Kataoka, T.; Kinoshita, H.; Kinoshita, S.; Iwamura, T.; Watanabe, S. Angew. Chem. Int. Ed. 2000, 39, 2358.

33. Patra, A.; Batra, S.; Joshi, B. S.; Roy, R.; Kundu, B.; Bhaduri, A. P. J. Org. Chem. 2002, 67, 5783.

34. Shi, M.; Jiang, J-K, Feng, Y. Org. Lett. 2000, 2, 2397.

35. Shi, M.; Feng, Y-S. J. Org. Chem. 2001, 66, 406.

36. Aggarwal, V. K.; Mereu, A.; Tarver, G. J.; McCague, R. J. Org. Chem. 1998, 63, 7183.

37. Kataoka, T.; Kinoshita, H.; Iwama, T.; Tsujiyama, S.-I.; Iwamura, T.; Watanabe, S.-I.; Muraoka, O.; Tanabe, G. Tetrahedron 2000, 56, 4725.

38. Kataoka, T.; Iwama, T.; Tsujiyama, S. Chem. Commun. 1998, 197.

39. Katritzky, A. R.; Manju, K.; Singh, S. K.; Meher, N. K. Tetrahedron 2005, 61, 2555 and reference therein

40. Katritzky, A. R.; Yannakopoulou, K.; Kumierkiewicz, W.; Aurrecoechea, J. M.; Palenik, G.J.; Koziol, A. E.; Szczesniak, M. J. Chem. Soc., Perkin Trans. 1 1987, 2673.

41. Katritzky, A. R.; Nair, S. K.; Qiu, G. Synthesis 2002, 199.

42. Katrizky, A. R.; Yannakopoulou, K.; Lue, P.; Rasala, D.; Urogdi, L. J. Chem. Soc., Perkin Trans. 1 1989, 225.

43. Katritzky, A. R.; Strah, S.; Belyakov, S. A. Tetrahedron 1998, 54, 7167.

44. Katrizky, A. R.; Abdel-Fattah, A. A. A.; Steel, P. J. Tetrahedron Lett. 2006, 47, 1465.

45. Katritzky, A. R.; Rachwal, S.; Rachwal, B. J. Chem. Soc., Perkin Trans. 1 1987, 805.

46. Azizi, N.; Saidi, M. R. Tetrahedron Lett. 2002,43, 4305.

47. Arend, M.; Westermann, B.; Risch, N. Angew. Chem. Int. Ed. 1998, 37, 1044 and references therein

48. Katritzky, A. R.; Pilarski, B; Urogdi, L. Org. Prep. Prod. Int. 1989, 21, 135.

49. Katritzky, A. R.; Rachwal, S.; Rachwal, B.; Steel, P. J. Org. Chem. 1992, 57, 4932. 
50. Boothe, J. E.; Flock, H. G.; Hoover, M. F. Macromol Sci. Chem .1970, A4, 1419.

51. Hoover, M. F. Macromol. Sci. Chem. 1970, A4, 1327.

52. Adrian, G.; Weber, D. Sciences Cimiques, Series C. 1971, 272, 1902.

53. Avci, D.; Osanyan, N.; Mathias, L.J. Polymer Preprints 2001, 42, 514.

54. Avci, D.; Mathias, L. J. Polymer Preprints 1999, 40, 784. 